\title{
The relative benefits of being optimistic: Optimism as a coping resource in multiple sclerosis and Parkinson's disease
}

\author{
Denise de Ridder*, Karlein Schreurs and Jozien Bensing \\ Department of Health Psychology, Utrecht University, The Netherlands
}

\begin{abstract}
Objective. To explore two issues involving the relationship between optimism and adaptation in chronically ill patients. The first issue involves the question whether the impact of optimism on adjustment is disease-specific, that is whether patients who are confronted with extreme levels of uncontrollability and unpredictability generated by their condition benefit more from an optimistic attitude than those who are confronted with less high levels of those disease characteristics. The second issue relates to the question whether greater optimism produces better adjustment or whether, in contrast, a medium level of optimism plays a more adaptive role.
\end{abstract}

Design. The cross-sectional design involves 166 patients with Parkinson's disease (PD) or multiple sclerosis (MS) recruited from patient's organizations.

Method. Regression analyses were employed to test for a disease-specific effect of dispositional optimism (measured by the Life Orientation Test) on coping (measured by the Coping Inventory for Stressful Situations) and adjustment (measured by the Sickness Impact Profile) by introducing interactions between optimism and disease in the analysis. Curvilinear effects were tested by introducing a squared optimism variable.

Results. These show that optimism has a disease-specific effect on physical adjustment and on the use of emotion-oriented and avoidant coping strategies. Although the study's cross-sectional design does not allow for causal inferences, these data suggest that MS patients may benefit more from optimism than PD patients and also that, in comparison with PD patients, optimism in MS patients does not necessarily promote avoidant and emotion-oriented coping. The results also show a medium level of optimism to promote the application of task-oriented and avoidant coping strategies.

Conclusion. It is concluded that context-specific constraints associated with type of disease may affect the assumed beneficial role of optimism on adjustment.

Chronic illness confronts patients with numerous threats and challenges: the need to preserve a reasonable emotional balance and a satisfactory self-image; the need to maintain a sense of competence and mastery; the need to deal with pain, fatigue, and other symptoms of the disease; and the need to prepare for an uncertain future (Moos \& 
Schaefer, 1984). In their efforts to manage these and the other stressors that the disease brings about, patients can rely on social and personal resources, including social support, financial resources, social skills, problem-solving skills, and positive beliefs which may facilitate the coping process (Lazarus \& Folkman, 1984). In the past few years, increasing attention has been devoted to positive beliefs as a coping assistance. The concept of positive beliefs refers to people framing their view of themselves, the world, and the future in positive terms. People with favourable self-beliefs and a positive outlook on life are generally healthier, in both a physical and a mental sense, and also more inclined to apply health-protecting behaviours than people with unfavourable self-beliefs (Peterson \& Bossio, 1991; Scheier \& Carver, 1992; Seligman, 1991).

Several approaches of the study of positive beliefs have been proposed. One of them is the dispositional optimism approach, which refers to the role of generalized expectancies for good outcomes (Scheier \& Carver, 1985). According to the model presented by Scheier and Carver (1985), dispositional optimism is assumed to reflect a willingness to persist when confronted with adversity. The positive effects of dispositional optimism on health can be accounted for by self-regulation theory, which states that individuals with positive expectations for the future are more likely to make an effort to reach their goals than those without such positive expectations. A rapidly growing body of empirical research has shown optimism to offer a fruitful perspective for explaining adaptation to a number of health problems (Scheier \& Carver, 1992), including such chronic diseases as cancer (Friedman et al., 1992), breast cancer (Carver et al., 1994), Parkinson's disease (Shifren, 1996) and rheumatoid arthritis (Brenner, Melamed, \& Panush, 1994). In particular, optimism appears to promote the use of problem-focused and approach strategies for coping, which are generally more beneficial than emotion-focused and avoidant strategies. For example, Scheier, Weintraub, and Carver (1986) found that dispositional optimism as measured by the Life Orientation Test was positively associated with a number of approach coping strategies (e.g. problem-focused coping, positive reappraisal) and negatively associated with avoidant strategies such as denial and distancing.

As studies of 'unrealistic optimism' have shown, positive beliefs are not always beneficial. Some people may tend to be overly optimistic and thereby underestimate their chances of becoming ill (Weinstein, 1982; Weinstein \& Klein, 1996). In this view, optimism pertains to a perception of personal invulnerability and undermines legitimate worry about health threats as demonstrated in a number of studies in the field of preventive medicine (Weinstein \& Klein, 1996). It has also been suggested that overly optimistic people are inclined towards wishful thinking, which can lead to a failure to take reasonable precautions and neglect of the actual level of personal distress (Cantor \& Norem, 1989).

To account for the differential impact of optimism on health and health behaviour, Schwarzer (1994) recently suggested that two kinds of optimism be distinguished: defensive or naïve optimism, which is an attempt to hide from real dangers with the perception that one is not at risk, and functional or 'cautious' optimism (cf. Wallston, 1994), which leads to vigilance and engagement in protective behaviours. Schwarzer formulated his distinction to account for the differential effects of optimism on general health problems, but the distinction may also be relevant for chronic disease. It may be argued, for example, that optimism is not only dysfunctional when it reflects an underestimation of health risks, but also when it leads to an inadequate appraisal of 
stressors and inadequate use of available coping resources. A typical naïve optimistic chronic patient, then, will engage in primarily avoidant coping strategies regardless of the demands of his or her disease. This is not to say that avoidant coping is always unhelpful. The hypothesis-of-fit approach advocated by Lazarus and Folkman (1984) suggests that adequate coping matches the demands of the stressful situation. That is, a cautious approach in which avoidant strategies alternate with approach strategies for coping - depending on the particular constraints of the situation-should provide the individual patient with the best opportunities for successfully adapting to his or her illness. Such an approach also suggests that the benefits of optimism may depend on the characteristics of the chronic disease, such as controllability, predictability and manageability. Although no direct empirical evidence is available, it can be argued that for controllable diseases, which heavily depend on adherence to a number of self-management techniques, being overly optimistic can be life-threatening. In contrast, for uncontrollable diseases, a reasonable degree of optimism may be necessary to make the effort needed to cope with various disease-related stressors.

As far as we know, none of the ideas generated by Schwarzer's distinction between functional/cautious optimism and defensive/naïve optimism have been tested empirically in chronically ill patients. Research on the role of optimism in chronic disease has rather been exclusively focused on the assumption that optimism is good, that greater optimism is better, and that optimism clearly enhances problem-focused coping and thereby fosters adaptation. This assumption is also shared by many patients who suffer from chronic diseases as they tend to idealize being optimistic and the associated behaviours of positive thinking and fighting the disease regardless of the actual problems they confront (de Ridder, Depla, Severens, \& Malsch, 1997). The extent to which chronic patients actually benefit from being optimistic remains unclear. The main goal of the present study is therefore to address the question of whether optimism is always helpful when suffering from chronic illness. In doing this, the study focuses on two separate issues.

The first issue relates to the idea that the nature and course of a disease may either enhance or limit the beneficial effects of optimism. That is, optimism may be an especially helpful resource when confronted with an illness that calls for perseverance and hope. Multiple sclerosis (MS) is an example of such a disease: it threatens people, especially women, at a young age when chronic illness is not expected. In addition, as medical textbooks point out, the symptoms and course of MS are characterized by a total lack of predictability and controllability and no (palliative) medical cure is available to ease its consequences (Paty \& Poser, 1984; Sibley, 1990). The variability of the course of MS is exemplified by some patients who have two or three exacerbations in a lifetime and never become disabled; at the other extreme are a few individuals who have frequent attacks, leading to death within a few years (Sibley, 1990). This medical account of disease characteristics is corroborated by patients who have repeatedly reported on the psychological burden created by the extreme unpredictability and uncontrollability of the course and symptoms of their disease (e.g. Brooks \& Matson, 1982; Foote, Piazza, Holcombe, Paul, \& Daffin, 1990; Murray, 1995). Although a significant number of MS patients suffer from depressive symptoms (Raos, Huber, \& Bornstein, 1992; Shubert \& Foliart, 1993), most patients have been found to engage in primarily positive strategies, such as positive thinking, humour and wishful thinking (Murray, 1995). In less extreme 
diseases, optimism may not have such a beneficial effect because it hinders acceptance of the illness and interferes with the application of appropriate coping behaviours. Parkinson's disease (PD), which is a progressive neurological condition characterized by tremors, rigidity and slowness of movement, is an example of the latter. Many PD patients suffer from depression (Raos et al., 1992) and, especially in the initial stages of the disease, optimism has been found to foster adaptation (Dakof \& Mendelsohn, 1989). As in the case of MS, the symptoms associated with PD interfere with such simple actions as walking and writing. In contrast to the case of MS, however, medication is available to manage the symptoms associated with PD, which makes the disease more controllable and less unpredictable than MS, both from a medical and a psychological point of view (Ellgring et al., 1993; Hoehn \& Yahr, 1967; Marr, 1991; Raos et al., 1992; Stern, 1988). Although no attempts have been made to quantify either the objective medical differences in controllability and unpredictability or the differences as they are experienced by patients suffering from these diseases, the study takes the reported difference in controllability and predictability as its starting point to explore whether any diseasedependent effects of optimism on adjustment exist. Such an approach appears appropriate considering the fact that medical opinions and lay views point in the same direction. The study also assumes that differences between diseases are larger than those within patients suffering from a particular disease because, although patients' perceptions of their illness have been found to affect adaptation, it has also been found that these perceptions are developed in close relationship with the reality of symptoms (Dakof \& Mendelsohn, 1989; de Ridder, Schreurs, \& Bensing, 1998; Felton \& Revenson, 1984; Heijmans \& de Ridder, 1998). As both MS and PD patients suffer from serious physical limitations caused by their disease, it is assumed that the variability of patients' responses is not larger than the differences between groups.

The second issue addressed pertains to the assumption that optimism may be dysfunctional when chronically ill people are overly optimistic and thereby neglect the constraints imposed upon their lives by the disease in question. This issue reflects the distinction between cautious vs. naïve optimism, as proposed by Schwarzer. It is assumed that naive optimists will report higher levels of (dispositional) optimism than cautious optimists and that naïve optimists simply expect favourable outcomes without taking further measures to make these outcomes happen. In contrast, cautious optimists or those patients with medium levels of optimism do not expect favourable outcomes to occur automatically, and are prepared to make an effort to obtain such goals (cf. Wallston, 1994). Put differently, it is assumed that on top of a disease-specific effect of optimism, curvilinear effect of optimism may be present and that those who engage in medium levels of optimism reach better adjustment than either low-optimistic or high-optimistic patients.

\section{Methods}

\section{Participants}

The participants were 70 patients suffering from PD and 96 patient suffering from MS. Both PD and MS patients were invited to participate in the study via a newsletter from the patients' organization. In the Netherlands, about $75 \%$ of MS patients and $15 \%$ of PD patients are members of the respective patients' organizations. About $10 \%(N=600)$ of the members of the MS patients' organization responded to the 
invitation and 96 of them were randomly selected for this study. Because of the self-referral procedure, it is suspected that more active members were more likely to participate in this study. In this sense, the results of the present study may not be representative of the views held by other patients suffering from MS. In the case of PD patients, the results are interpreted with even more caution since only a selected group of patients $(15 \%, N=5000)$ are members of the PD patients' organization, of whom 115 responded to the invitation. Eventually, 70 PD patients were included in the present study on optimism. The limitations of the recruitment procedure are discussed in more detail in the Discussion section.

The 70 patients with PD ( $46 \%$ women) were aged 45 to 75 years (mean age $=62.4$ years, SD = 8.2). Most of the patients (77\%) were married. Because of their age and their illness, only $7 \%$ had paid employment. The disease characteristics of the patients with PD were measured by asking about illness duration $(M=12.5$ years, $\mathrm{SD}=8.2$ ) and the number of symptoms the patients experienced, such as stiffness, slowness of movement, tremors and speech impediments $(M=4.4$ symptoms on a $1-10$ number of symptoms scale, $\mathrm{SD}=1.7)$. All of the patients used medication to control their disease (in particular, levodopa-preparations, dopamine agonists and selegiline), and only $46 \%$ received treatment other than medical treatment (in particular, physical therapy). A minority of the patients ( $8 \%$ ) had also undergone (stereotactic) surgery.

Of the $96 \mathrm{MS}$ patients, $76 \%$ were women, which is significantly more than for the PD patients $(p<.001)$. The MS patients were also significantly $(p<.001)$ younger than the PD patients (mean age $=45.2$ years, $\mathrm{SD}=9.4$, range $=19-68)$. Of the MS patients, $76 \%$ were married and $17 \%$ still had paid employment. The patients reported suffering from $M S$ for a large number of years $(M=15.1$ years, $\mathrm{SD}=8.9)$. The mean number of symptoms that the patients experienced was 3.7 (on a scale of 1 to 10) with the symptoms of fatigue, speech impediment and partial paralysis most frequently reported. Only $34 \%$ of the MS patients used medication and almost all of them received no other treatment than regular neurological check-ups.

\section{Measures}

All measures were administered by means of a postal self-report questionnaire.

Optimism was measured using the Life Orientation Test (Scheier \& Carver, 1985), which consists of eight items (and four filler items). Instead of reverse scoring the negative items, as recommended in the original test, the experimenters followed Marshall's suggestion (Marshall, Wortman, Kusulas, Hervig, \& Vickers, 1992) of differentiating between an optimism score (four items, Cronbach's $\alpha .78$ for PD patients and .75 for MS patients) and a pessimism score ( 4 items, Cronbach's $\alpha .78$ for PD patients and .65 for MS patients). ${ }^{1}$ The scores on this test could range from 1 (I disagree a lot) to 5 (I agree a lot), with higher scores indicating more optimism (or pessimism). For both the PD and the MS patients, optimism and pessimism were found to be weakly and negatively related $(-.28, p<.01$ and $-.34, p<.001$ respectively). When compared to the internal consistency of the single scales, these correlations are relatively weak and support the experimenters' decision to distinguish separate scales for optimism and pessimism (cf. Hummer, Dember, Melton, \& Schefft, 1992).

Coping was assessed using the Coping Inventory for Stressful Situations, which was developed by Endler and Parker (1994). The psychometric properties of this test are good with high internal consistencies and a high test-retest reliability. Endler and Parker (1994) have also reported extensive external validity data on the CISS. For example, the patterns of correlations between the CISS and the Ways of Coping Questionnaire developed by Lazarus and Folkman converged and diverged in a theoretically meaningful way. The 48 items represent three coping styles (with 16 items each and sufficient internal consistencies): task-oriented coping (Cronbach's $\alpha .89$ for PD patients and .90 for MS patients), emotion-oriented coping (Cronbach's $\alpha .82$ for PD patients and .90 for MS patients), and avoidant coping (Cronbach's $\alpha .78$ for PD patients and .79 for MS patients). The scales are weakly related with positive correlations between task coping and emotion coping $(.26, p<.01$ in MS patients; .26 , n.s. in PD patients) and task coping and avoidant coping (.22, n.s. in MS patients, $.35, p<.01$ in PD patients). Emotion and avoidant coping strategies were not found to be related for either patient group $(r=.04$, n.s.).

Depression was measured using the Beck Depression Inventory (21 items, Cronbach's $\alpha .80$ for PD patients, .86 for MS patients), with higher scores indicating more depressive feelings.

\footnotetext{
${ }^{1}$ Principal components analysis of the eight LOT-items revealed two factors with eigenvalues $>1.5$ in both patient groups, accounting for $56 \%$ of the variance in MS patients and $57 \%$ of the variance in PD patients.
} 
Quality of life was measured using the Sickness Impact Profile (SIP; Bergner, Bobbitt, Carter, \& Gilson, 1981), which is a self-report measure of several aspects of daily functioning and provides a particularly reliable picture of behavioural changes as a consequence of health status and has also proven to be a useful instrument for discriminating between patient groups. In the present study, three scales from the shortened version of the SIP were used: physical autonomy (17 patients, Cronbach's $\alpha .82$ for PD patients and .87 for MS patients), social functioning (12 items, Cronbach's $\alpha .75$ for PD patients and .78 for MS patients), and psychological functioning (6 items, Cronbach's $\alpha .75$ for both PD and MS patients). Higher scores indicate better adjustment. The correlations between the scales were found to be moderate (ranging from .30 to .40 for the social functioning scale with the physical autonomy scale and the psychological functioning scale). The correlations between physical autonomy and psychological functioning were found to be very weak for both of the patient groups $(r<.10)$.

\section{Results}

Descriptive statistics are presented in Table 1, where it can be seen that the PD and MS patients do not differ in their self-reported levels of optimism and pessimism and that both report rather similar levels of optimism as have been reported earlier on patients suffering from chronic disease (cf. Carver et al., 1994; Friedman et al., 1992; Shifrin, 1996; Tennen et al., 1994). Neither the PD nor the MS patients report intense feelings of depressed mood as measured by the BDI. The PD and the MS patients engage in the three different ways of coping in a similar fashion and report more task-oriented coping than avoidant coping and emotion-oriented coping ( $t$ test paired samples, $p<.001$ for both PD and MS patients). The level of adjustment was also found to be similar for the patient groups with both the PD and MS patients reporting rather high levels of physical autonomy and psychological adjustment, but a rather low level of social adjustment ( $t$ test paired samples, $p<.001$ for both PD and MS patients).

Table 1. Means and standard deviations for PD $(N=70)$ and MS $(N=96)$ patients

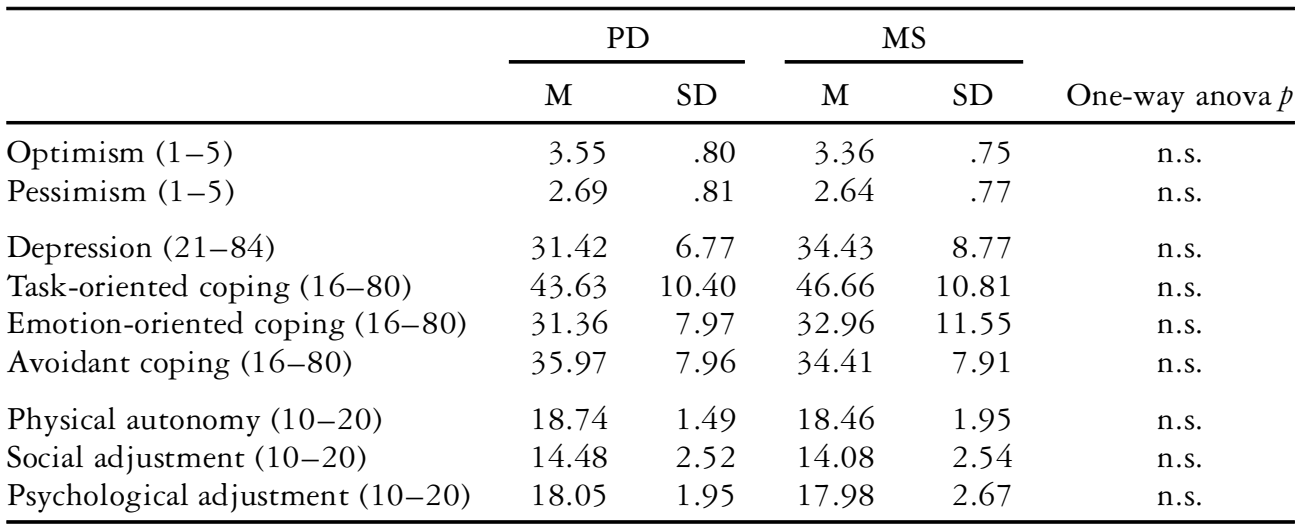

In Table 2, the correlations between optimism/pessimism and particular demographic characteristics, coping styles and quality of life for the PD and MS patients are presented. In neither patient group was optimism or pessimism found to be related to demographic characteristics. Optimism and pessimism generally relate to other variables in the opposite direction, but with a similar strength. Discussion is therefore focused on the 
Table 2. Pearson correlations for optimism and pessimism with other variables in PD $(N=70)$ and MS $(N=96)$ patients

\begin{tabular}{lccccc}
\hline & \multicolumn{2}{c}{ PD } & & \multicolumn{2}{c}{ MS } \\
\cline { 2 - 3 } \cline { 5 - 6 } \cline { 5 - 6 } & Optimism & Pessimism & & Optimism & Pessimism \\
\hline Sex & -.03 & -.07 & & .03 & .03 \\
Age & -.19 & .19 & & .06 & -.03 \\
Marital status & .03 & -.11 & & .09 & .13 \\
Paid employment & -.09 & .18 & & -.11 & .15 \\
Illness duration & .18 & .10 & & .03 & -.06 \\
Depression & -.19 & $.32^{*}$ & & $-.47 * *$ & $.53^{* *}$ \\
Task-oriented coping & .25 & $-.42^{* *}$ & & -.03 & .02 \\
Emotion-oriented coping & -.23 & .18 & & $-.53^{* *}$ & $.58^{* *}$ \\
Avoidant coping & $.30^{*}$ & -.20 & & .13 & -.07 \\
Physical autonomy & -.13 & -.15 & & $.35^{* *}$ & -.14 \\
Social adjustment & .20 & $-.38^{*}$ & & $.28^{*}$ & $-.26^{*}$ \\
Psychological adjustment & $.27 *$ & $-.33^{*}$ & & $.46^{* *}$ & $-.39^{* *}$ \\
\hline
\end{tabular}

$* p<.01 ; * p<.001$.

associations between optimism and adjustment variables. Interestingly, when these associations are considered, two disease-specific patterns are suggested. In the case of PD patients, optimism is not significantly related to either coping or adjustment except in the case of avoidant coping and psychological adjustment: optimistic PD patients tend to adopt avoidant coping strategies more frequently than their less optimistic counterparts and they appear to be more psychologically adjusted. In the case of MS patients, higher levels of optimism appear to be related to less depression and higher levels of physical, social and psychological adjustment. To explore the suggested disease-specific pattern the experimenters tested in a multivariate model whether significant differences exist between PD and MS patients in the way optimism affects coping and adjustment. In addition, they simultaneously tested for a potential curvilinear effect of optimism.

In Table 3, the results of three hierarchial regression analyses with each aspect of adjustment as a dependent variable are presented. The hierarchy of steps parallels the experimenters' hypothetical model involving a differential disease-dependent effect of optimism on adjustment. The first step controls for the impact of disease by introducing a dummy variable representing the particular diagnosis (i.e. PD or MS). The second step controls for any sex or age differences between the patient groups. Also controlled for were the level of depressive mood and pessimism to provide a more critical test of the role of optimism. In the third step, the coping resources (optimism and coping styles) are introduced. Finally, in the fourth step, an interaction term representing the diseasedependent impact of optimism is introduced. The fourth step also involves the introduction of the squared optimism to test for a potential curvilinear effect. Put in statistical terms, the question is whether a linear effect of more optimism leading to better adjustment or a curvilinear effect of optimism with an optimal (instead of a maximum) level leading to better adjustment is found. In order to model an inverted U-shaped curvilinear effect, the experimenters computed a squared optimism variable (after 
mean-centering the optimism variable) and then inverted it by multiplying with -1 . Next, this squared variable was introduced in the regression analyses together with the disease-specific optimism variable.

Table 3. Prediction of physical autonomy, social adjustment and psychological adjustment from a context-specific optimism model $(N=166)$. Beta are reported per step

\begin{tabular}{|c|c|c|c|c|c|c|}
\hline \multirow[b]{2}{*}{ Dependent } & \multicolumn{2}{|c|}{ Physical autonomy } & \multicolumn{2}{|c|}{ Social adjustment } & \multicolumn{2}{|c|}{$\begin{array}{l}\text { Psychological } \\
\text { adjustment }\end{array}$} \\
\hline & $\beta$ & $\begin{array}{l}R^{2} \text { per } \\
\text { step }\end{array}$ & $\beta$ & $\begin{array}{l}R^{2} \text { per } \\
\text { step }\end{array}$ & $\beta$ & $\begin{array}{l}R^{2} \text { per } \\
\text { step }\end{array}$ \\
\hline Step 1 & & $0 \%$ & & $0 \%$ & & $0 \%$ \\
\hline $\begin{array}{l}\text { Diagnosis } \\
\text { Step } 2\end{array}$ & -.03 & $10 \%$ & -.06 & $12 \%$ & .03 & $25 \%$ \\
\hline depression & -.14 & & $-.17 *$ & & $-.35 * * *$ & \\
\hline sex & .03 & & -.06 & & -.02 & \\
\hline age & $-.35 * * *$ & & -.15 & & .11 & \\
\hline pessimism & -.06 & & $-.23 * *$ & & $-.24 * * *$ & \\
\hline Step 3 & & $18 \%$ & & $16 \%$ & & $42 \%$ \\
\hline optimism & .10 & & .08 & & .12 & \\
\hline task-oriented coping & $.31 * * *$ & & .09 & & $.23 * * *$ & \\
\hline emotion-oriented coping & $-.19 *$ & & -.07 & & $-.44 * * *$ & \\
\hline avoidant coping & -.01 & & .11 & & .02 & \\
\hline Step $4^{b}$ & & $20 \%$ & & $17 \%$ & & $43 \%$ \\
\hline IR optimism & $.22 * * *$ & & .04 & & .09 & \\
\hline SQ optimism & -.01 & & -.11 & & -.04 & \\
\hline Total Adj. $R^{2}$ & & $14 \% * * *$ & & $11 \% * *$ & & $38 \% * * *$ \\
\hline
\end{tabular}

$* p<.05 ; * * p<.01 ; * * * p<.001$.

${ }^{a}$ Dummy variable $0=\mathrm{PD}, 1=\mathrm{MS}$.

${ }^{b}$ Interaction terms of illness-related (IR) variable (i.e. the product of variable and dummy). Squared (SQ) variables were added to model a curvilinear effect of optimism.

As can be seen in Table 3, a main effect of disease is absent. After controlling for sex, age, depression and pessimism, it appears that coping resources significantly account for a large portion of the variance in adjustment and in psychological functioning in particular. Noted, however, that the impact of optimism is non-significant when compared to the impact of coping variables (as can be inferred from the beta weights presented in Table 3). In addition to the main effect of coping resources, the interaction term involving the dummy disease variable with optimism was found to significantly contribute to the explanation of physical autonomy. This suggests a significant disease-dependent effect of optimism on this aspect of adjustment: optimism appears to have a stronger positive impact on the physical adjustment of MS patients when compared to PD patients (see Fig. 1). A curvilinear effect of optimism appears to be absent in the prediction of physical, psychological, and social adjustment both for PD and MS patients.

In Table 4, a similar approach to that adopted for the prediction of adjustment is applied for the prediction of coping. The effects of optimism and its possible illnessdependent and curvilinear effects are examined after controlling for diagnosis in the first 


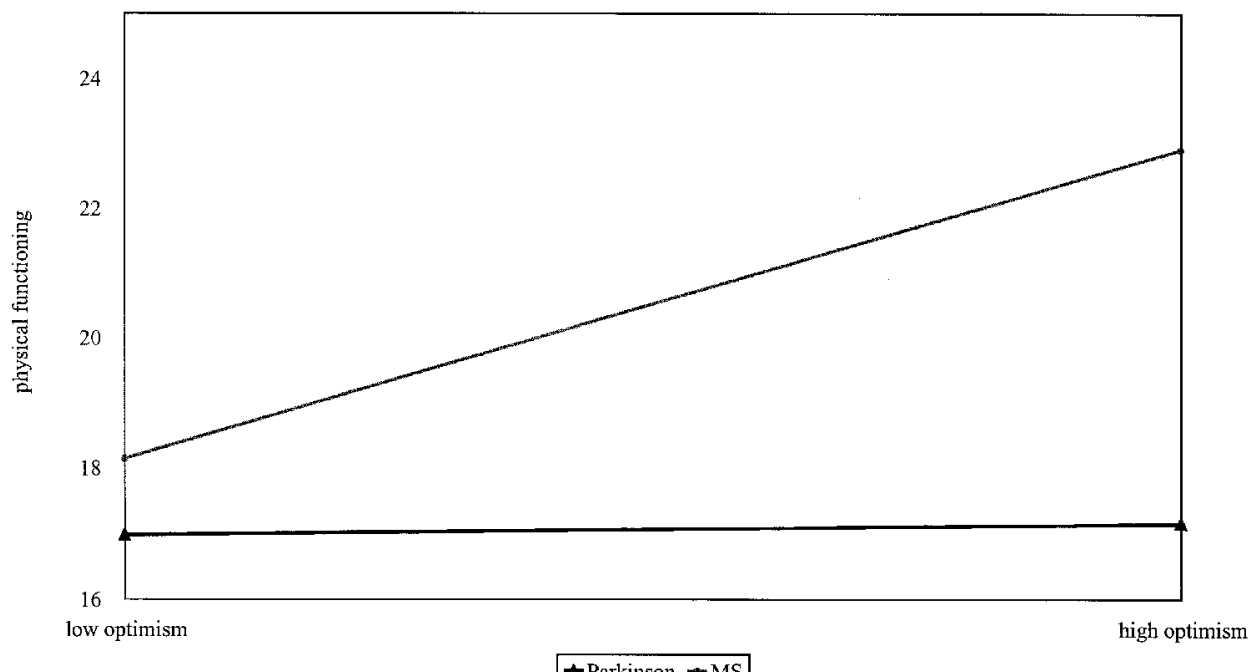

Figure 1. Interaction of optimism and diagnosis on physical functioning.

step and for sex, age, depressive mood and pessimism in the second step. As can be seen, task-oriented coping and avoidant coping are rather poorly predicted by the model. Nevertheless, both in avoidant and in emotion-oriented coping a significant main effect of optimism is present, showing that a high level of optimism encourages avoidant coping but hinders emotion-oriented coping. Remarkably, optimism does not seem to contribute to task-oriented coping. Instead, the significant main effect pessimism (step 2) demonstrates that task-oriented coping is hindered by a high level of pessimism. Noted also that, while pessimism appears to hinder task-oriented coping, depressive mood appears to promote it. The introduction of an interaction term representing an illnessdependent impact of optimism in the final step improves the model significantly in the case of emotion-oriented coping, showing that this type of coping is affected by optimism more strongly in the case of MS patients. That is, when compared to their PD counterparts, optimistic MS patients do not necessarily employ as many emotion-focused coping strategies (see Fig. 2). In contrast, an illness-dependent effect of optimism appears to be absent in the case of task-oriented and avoidant coping. These latter types of coping appear to be associated with medium instead of a high level of optimism. These data strongly suggest that medium rather than high levels of optimism contribute to the employment of task-oriented and avoidant coping strategies, both in case of MS and PD patients. Such a curvilinear pattern can also be detected when comparing the means of task-oriented coping and avoidant coping for patients with low, medium and high levels of optimism: medium optimistic patients report higher levels of task-oriented coping and avoidant coping ( $M=2.94$ and $M=2.30$ respectively) than either low optimistic patients $(M=2.54$ and $M=2.00$ respectively or high optimistic patients $(M=2.85$ and $M=2.14$ respectively), although it must be stated also that especially in the case of task-oriented coping, medium and high optimistic patients employ a relatively similar amount of this particular coping strategy (see Figs. 3 and 4). 
Table 4. Prediction of task-oriented, emotion-oriented, and avoidant coping from a contextspecific optimism model $(N=166)$. Betas are reported per step

\begin{tabular}{|c|c|c|c|c|c|c|}
\hline \multirow[b]{2}{*}{ Dependent } & \multicolumn{2}{|c|}{ Task-oriented coping } & \multicolumn{2}{|c|}{$\begin{array}{l}\text { Emotion-oriented } \\
\text { coping } \\
\end{array}$} & \multicolumn{2}{|c|}{ Avoidant coping } \\
\hline & $\beta$ & $R^{2}$ per step & $\beta$ & $R^{2}$ per step & $\beta$ & $R^{2}$ per step \\
\hline $\begin{array}{l}\text { Step } 1 \\
\text { diagnosis }^{a}\end{array}$ & .13 & $2 \%$ & .07 & $1 \%$ & -.09 & $1 \%$ \\
\hline $\begin{array}{l}\text { Step } 2 \\
\quad \text { depression } \\
\text { sex } \\
\text { age } \\
\text { pessimism }\end{array}$ & $\begin{array}{l}.20 * * \\
.02 \\
-.02 \\
-.20 * *\end{array}$ & $7 \%$ & $\begin{array}{l}.40 * * * \\
.08 \\
-.17 * \\
.30 * * *\end{array}$ & $37 \%$ & $\begin{array}{l}-.02 \\
.26 * * \\
-.15 \\
-.10\end{array}$ & $10 \%$ \\
\hline $\begin{array}{l}\text { Step } 3 \\
\text { optimism }\end{array}$ & .10 & $8 \%$ & $-.23 * * *$ & $42 \%$ & $.18 *$ & $12 \%$ \\
\hline $\begin{array}{l}\text { Step } 4^{b} \\
\text { IR optimism } \\
\text { SQ optimism }\end{array}$ & $\begin{array}{l}-.02 \\
.19 * *\end{array}$ & $13 \%$ & $\begin{array}{l}-.20 * * \\
-.04\end{array}$ & $43 \%$ & $\begin{array}{l}-.06 \\
.18 *\end{array}$ & $16 \%$ \\
\hline Total Adj. $R^{2}$ & & $9 \% *$ & & $40 \% * * *$ & & $12 \% * *$ \\
\hline
\end{tabular}

$* p<.05 ; * * p<.01 ; * * * p<.001$.

${ }^{a}$ Dummy variable $0=\mathrm{PD}, 1=\mathrm{MS}$.

${ }^{b}$ Interaction terms of illness-related (IR) variables (i.e. the product of variable and dummy). Squared (SQ) variables were added to model a curvilinear effect of optimism.

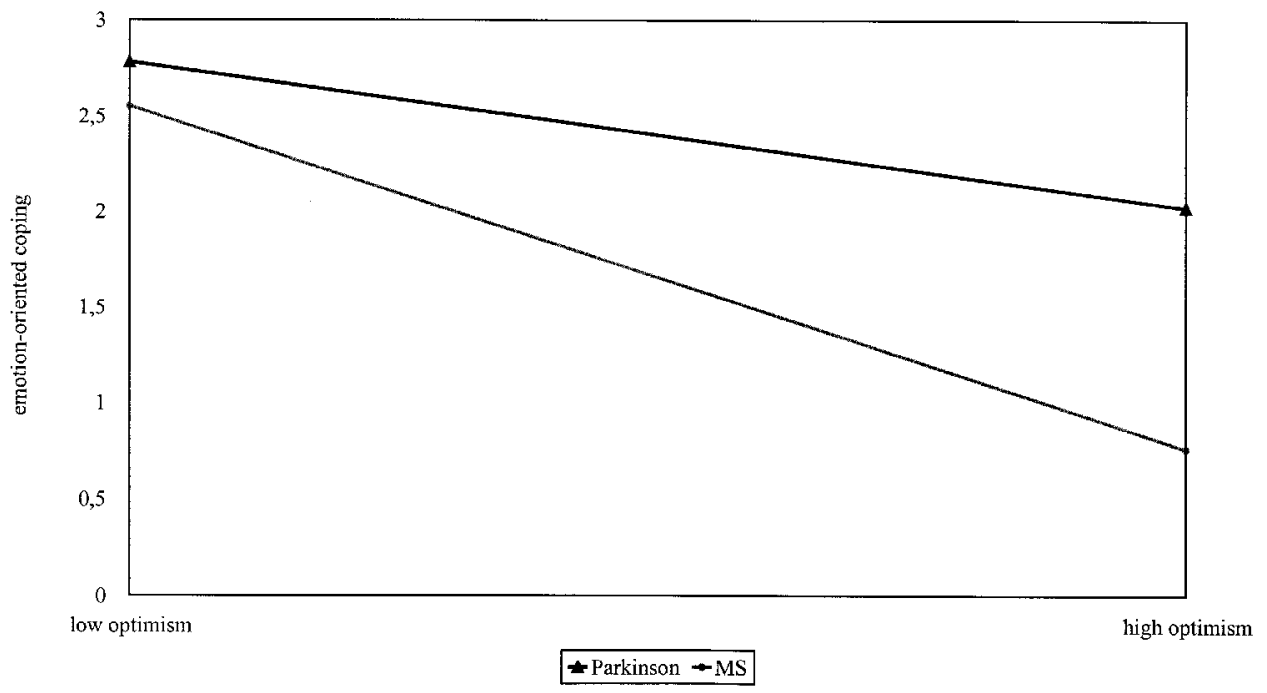

Figure 2. Interaction of optimism and diagnosis on emotion-oriented coping. 


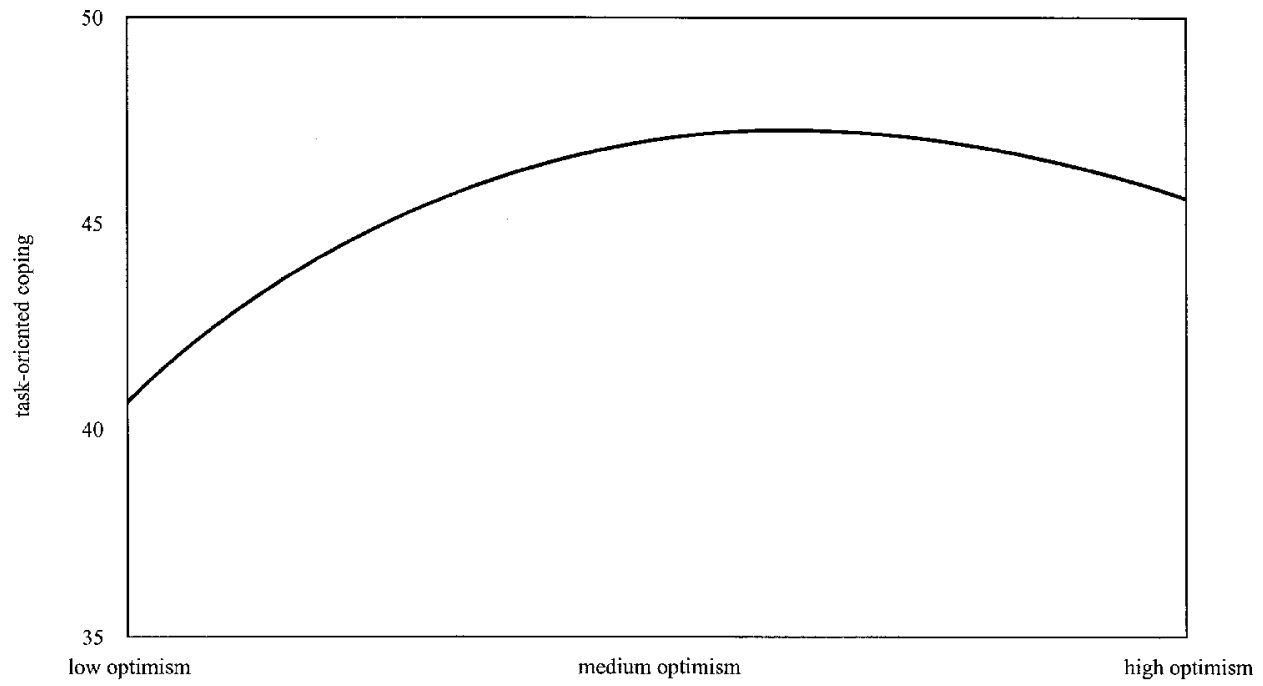

Figure 3. Relation of optimism and task-oriented coping.

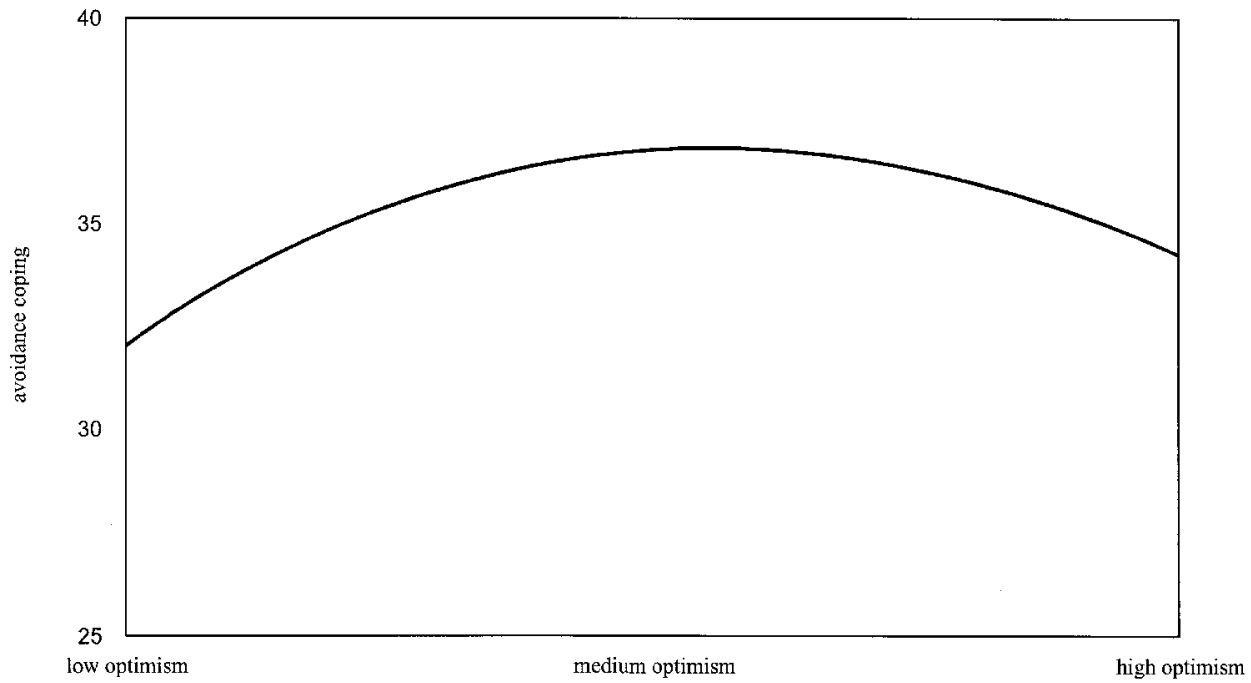

Figure 4. Relation of optimism and avoidance coping. 


\section{Discussion}

The study shows MS and PD patients to be fairly optimistic, although a considerable number of the patients report either low optimism (14\% scored more than one standard deviation below the scale mean) or high pessimism (20\% scored one or more standard deviation above the scale mean). The average optimism scores for the MS $(M=3.36)$ and PD $(M=3.55)$ patients resemble those for patients suffering from such other chronic diseases as Kahler's disease, Addison's disease, Cushing's Syndrome and Chronic Fatigue Syndrome (Fournier \& de Ridder, 1997). The findings of the present study nevertheless suggest that the actual impact of optimism on adjustment may to some extent be illness-dependent. In addition, the data show that greater optimism does not automatically lead to better ways of coping with chronic illness. Before discussing these findings in greater detail, the difference between optimism and pessimism is briefly considered.

Although factor analysis and the low correlations between optimism and pessimism suggest distinct dimensions, the patterns of correlations for optimism and pessimism with other variables reveal some similarities between the concepts. Clear effects of both optimism and pessimism on coping strategies can be seen while their impact on adjustment is almost absent. Closer examination shows pessimism to promote emotion-oriented coping in particular and optimism to block such coping. In addition, optimism helps patients engage in avoidant coping. These findings contradict those reported by Carver and Scheier, who have repeatedly pointed to the plausibility of a strong association between optimism and active problem-oriented behaviour. Of course, the different results may be because of the particular characteristics of patients who have been suffering from chronic illness for a rather long period of time. With these remarks in mind, also the role of depressive mood is noteworthy. The data suggest that although depressive mood generally hinders adjustment, it also encourages task-oriented and emotion-oriented coping. This remarkable finding may be interpreted in a fashion that depressive mood implies a more 'realistic' view on life and therefore more engagement in these 'approach'-types of coping. Further research should be undertaken.

The issue of whether optimism has an illness-dependent effect is now discussed. When the two patient groups are compared, optimism is found to have a stronger impact in the case of MS: an illness-dependent effect of optimism is found for physical autonomy and emotion-oriented coping. MS patients appear to profit more from optimism than PD patients, which suggests that optimism leads to better adjustment and less preoccupation with emotions as a result of being ill in the case of MS. Although the researchers have no detailed information on the manner in which optimism relates to adaptation, it may be that MS patients benefit more from optimism because it is their only hope, and that PD patients benefit less from optimism because a more realistic approach is needed for adjustment. Optimism does not protect one from the course of PD and may even hinder appropriate action at some times. Noted also that PD patients appear less able to protect themselves from depression by being optimistic, which most likely stems from the biological underpinnings of depression in PD.

In the present exploration of the impact of optimism, the researchers also hypothesized that high levels of optimism may not always be beneficial and that such high levels of optimism may even reflect someone ignoring the reality of being ill and a failure to take 
the measures needed to deal with the illness. The findings suggest that medium levels of optimism promote higher levels of task-oriented coping and avoidant coping, which means a curvilinear effect of optimism on these types of coping. This was not the case for emotion-oriented coping where high levels of optimism and the associated absence of negative emotions clearly produced less emotion-focused coping. The non-linear effect of optimism pertains to coping strategies in particular and thus supports the research of Cantor (Cantor \& Norem, 1989), who suggests 'defensive pessimists' or those who do not hold positive expectations but are more motivated to make an effort to accomplish the best are better adjusted than 'real optimists'. No evidence for an inverted U-shaped effect of optimism on adjustment was found, which suggests that the role of optimism as a coping resource may be more complex than suggested by the work on dispositional optimism (cf. Maes, Leventhal, \& de Ridder, 1996). In this view, optimism is assumed to lead to active problem-focused behaviour and thereby better adjustment while being a chronically ill patient, faced with an uncertain future and uncontrollable symptoms, challenges this assumption. A medium level of optimism may offer a more fruitful approach to being chronically ill and, when the disease can be controlled to some extent, it may even be wiser to not rely on optimism alone and turn to other coping resources for adjusting to the illness.

Of course, the limitations of this study should be kept in mind. The sample size was rather small and because of the self-referral procedure a biased picture may have been created, especially concerning PD patients. It is likely that members of a patients' organization who apply actively to participate in a study are also those patients who cope with disease in a more active way. In addition, it is unlikely that severely depressed patients react on an advertisement in a journal. Thus, the sample of this study probably consists of patients who are adapting relatively well to their ailment. Another limitation of the study concerns the cross-sectional design not allowing for establishing causal relationships which may be theoretically relevant. This remark especially concerns modelling of the effects of optimism on either coping or adjustment. Although it appears to be theoretically plausible, the experimenters have not chosen to test whether optimism may affect adjustment mediated by coping. The choice to focus on the assessment of disease-dependent and/or curvilinear effects of optimism does not imply that indirect effect of optimism via coping would not be plausible too. Further research concerning this aspect is recommended. Despite these concerns, it is believed that the results of this exploratory study suggest that one should exercise caution about the assumed ever-present beneficial effect of optimism, regardless of the type of illness patients are dealing with. Further research should clarify under which conditions positive expectations overrule the constraints imposed on patients by the nature of their disease (Fournier, de Ridder, \& Bensing, 1999).

\section{References}

Bergner, M., Bobbitt, R. A., Carter, W. B., \& Gilson, B. S. (1981). The Sickness Impact Profile: Development and final revision of a health status measure. Medical Care, 19, 787-805.

Brenner, G. F., Melamed, B. G., \& Panush, R. S. (1994). Optimism and coping as determinants of psychosocial adjustment to rheumatoid arthritis. Journal of Clinical Psychology in Medical Settings, 1, 115-134.

Brooks, N. A., \& Matson, R. R. (1982). Social-psychological adjustment to multiple sclerosis: A longitudinal study. Social Science and Medicine, 16, 2129-2135. 
Cantor, N., \& Norem, J. K. (1989). Defensive pessimism and stress and coping. Social Cognition, 7, 92-112. Carver, C. S., Pozo-Kaderman, C., Harris, S. D., Noriega, V., Scheier, M. F., Robinson, D. S., Ketcham, A. S., Moffat, F. L., \& Clark, K. C. (1994). Optimism versus pessimism predicts the quality of women's adjustment to early stage breast cancer. Cancer, 73, 1213-1220.

Dakof, G. A., \& Mendelsohn, G. A. (1989). Patterns of adaptation to Parkinson's disease. Health Psychology, 8, $355-372$.

de Ridder, D., Depla, M., Severens, P., \& Malsch, M. (1997). Beliefs on coping with illness: A consumer's perspective. Social Science and Medicine, 44, 553-559.

de Ridder, D., Schreurs, K., \& Bensing, J. (1998). Adaptive tasks, coping and quality of life of chronically ill patients: The case of Parkinson's disease and Chronic Fatigue Syndrome. Journal of Health Psychology, 3, 87-101.

Ellgring, H., Seiler, S., Nagel, U., Perleth, B., Gasser, T., \& Oertel, W. H. (1990). Psychosocial problems of Parkinson patients: Approaches to assessment and treatment. Advances in Neurology, 53, 349-353.

Endler, N. S., \& Parker, D. A. (1994). Assessment of multidimensional coping: Task, emotion, and avoidance strategies. Psychological Assessment, 6, 50-60.

Felton, B. J., \& Revenson, T. A. (1984). Coping with chronic illness: A study of illness controllability and the influence of coping strategies on psychological adjustment. Journal of Consulting and Clinical Psychology, $52,343-353$.

Foote, A. W., Piazza, d. Holcombe, J., Paul, P., \& Daffin, P. (1990). Hope, self-esteem and social support in persons with Multiple Sclerosis. Journal of Neuroscience and Nursing, 22, 155-159.

Fournier, M., \& de Ridder, D. (1997). Optimism and chronic illness. Department of Clinical Psychology and Health Psychology, Utrecht University, unpublished manuscript.

Fournier, M., de Ridder, D., \& Bensing, J. (1999). Optimism and adaptation to Multiple Sclerosis: What does optimism mean? Journal of Behavioral Medicine, 22, 303-326.

Friedman, L. C., Nelson, D. V., Baer, P. E., Lane, M., Smith, F. E., \& Dworkin, R. J. (1992). The relationship of dispositional optimism, daily life stress, and domestic environment to coping methods used by cancer patients. Journal of Behavioral Medicine, 15, 127-141.

Heijmans, M., \& de Ridder, D. (1998). Assessing illness representations in chronic illness-explorations of their disease-specific nature. Journal of Behavioral Medicine, 21, 485-503.

Hoehn, M. M., \& Yahr, M. D. (1967). Parkinsonism: Onset, prog ression, and morbidity, Neurology, 17, 427-442. Hummer, M. K., Dember, W. N., Melton, R. S., \& Schefft, B. K. (1992). On the partial independence of optimism and pessimism. Current Psychology, Research and Reviews, 11, 37-50.

Lazarus, F. S., \& Folkman, S. (1984). Stress, appraisal and coping. New York: Springer.

Maes, S., Leventhal, H., \& de Ridder, D. (1996). Coping with chronic diseases. In M. Zeidner \& N. S. Endler (Eds.), Handbook of coping: Theory, research, applications (pp. 221-251). New York: Wiley.

Marr, J. (1991). The experience of living with Parkinson's disease. Journal of Neuroscience and Nursing, 23, $325-330$.

Marshall, G. N., Wortman, C. B., Kusulas, J. W., Hervig, L. K., \& Vickers, R. R. (1992). Distinguishing optimism from pessimism: Relations to fundamental dimensions of mood and personality. Journal of Personality and Social Psychology, 62, 1067-1074.

Moos, R. H., \& Schaefer, J. A. (1984). The crisis of physical illness. In R. H. Moos (Ed.), Coping with physical illness: Vol. 2. New perspectives (pp. 3-25). New York: Plenum.

Murray, T. J. (1995). The psychosocial aspects of multiple sclerosis. Neurologic Clinics, 13, 197-223.

Paty, D. W., \& Poser, C. M. (1984). Clinical symptoms and signs of multiple sclerosis. In C. M. Poser (Ed.), The diagnosis of multiple sclerosis (pp. 27-43). New York: Thieme-Stratton.

Peterson, C., \& Bossio, L. M. (1991) Health and optimism: New research on the relationship between positive thinking and physical well-being. New York: Free Press.

Raos, M., Huber, S. J., \& Bornstein, R. A. (1992). Emotional changes with multiple sclerosis and Parkinson's disease. Journal of Consulting and Clinical Psychology, 50, 369-378.

Scheier, M. F., \& Carver, C. S. (1985). Optimism, coping, and health: Assessment and implications of generalized outcome expectancies. Health Psychology, 4, 219-247.

Scheier, M. F., \& Carver, C. S. (1992). Effects of optimism on psychological and physical well-being: Theoretical overview and empirical updat. Cognitive Therapy and Research, 16, 201-228.

Scheier, M. F., Weintraub, J. K., \& Carver, C. S. (1986). Coping with stress: Divergent strategies of optimists and pessimists. Journal of Personality and Social Psychology, 51, 1257-1264. 
Schwarzer, R. (1994). Optimism, vulnerability, and self-beliefs as health-related cognitions: A systematic overview. Psychology and Health, 9, 161-180.

Seligman, M. E. P. (1991). Learned optimism. New York: Knopf.

Shifren, K. (1996). Individual differences in the perception of optimism and disease severity: A study among individuals with Parkinson's disease. Journal of Behavioral Medicine, 19, 241-271.

Shubert, D. S., \& Foliart, R. H. (1993). Increased depression in multiple sclerosis patients: A meta-analysis. Psychosomatics, 34, 124-130.

Sibley, W. A. (1990). Diagnosis and course of Multiple Sclerosis. In S. M. Rao (Ed.), Neurobehavioral aspects of multiple sclerosis (pp. 5-14). Oxford: Oxford University Press.

Smith, R. W., Pope, M. K., Rhodewalt, F., \& Poulton, J. L. (1989). Optimism, neuroticism, coping and symptom reports: An alternative interpretation of the life orientation test. Journal of Personality and Social Psychology, 56, 640-648.

Stern, M. B., \& Hurtig, H. I. (1999). Parkinson's disease and Parkinsonian syndromes. Philadelphia: Saunders.

Tennen, H., \& Affleck, G. (1987). The costs and benefits of optimistic explanations and dispositional optimism. Journal of Personality, 55, 377-393.

Wallston, K. A. (1994). Cautious optimism vs. cockeyed optimism. Psychology and Health, 9, 201-203.

Weinstein, N. D. (1982). Unrealistic optimism about susceptibility to health problems. Journal of Behavioral Medicine, 5, 441-460.

Weinstein, N. D., \& Klein, W. M. (1996). Unrealistic optimism: Present and future. Journal of Social and Clinical Psychology, 15, 1-8. 